\title{
Alimentação de porcas gestantes e lactantes com dietas contendo saponinas
}

\author{
Feeding sows in gestation and lactation with diets containing saponins
}

\author{
Lucélia Hauptli ${ }^{1}$ Paulo Alberto Lovatto
}

\section{RESUMO}

Foi realizado um experimento para avaliar o desempenho de porcas e suas leitegadas alimentadas com dietas que continham saponinas. Trinta porcas geneticamente homogêneas foram distribuídas em dois tratamentos, dieta testemunha e dieta testemunha com adição de 160ppm de fontes de saponinas. $O$ delineamento utilizado foi o de blocos ao acaso, tendo como fator de bloqueamento a ordem de parto. Nas porcas, foram avaliados: o consumo de ração, características das fezes (cor e textura) e escore corporal. Nos leitões, foram avaliados: número de nascidos vivos, nascidos mortos, mumificados; pesos ao nascer e ao desmame e mortalidade na lactação. Não foram encontradas diferenças na cor das fezes das fêmeas. Na textura, as fezes das fêmeas que receberam a dieta com a adição de saponinas foram cerca de $11 \%$ mais duras $(P<0,05)$. Na última semana de lactação, as fêmeas alimentadas com a adição de saponinas apresentaram um escore corporal $12 \%$ superior $(P<0,05)$ às fêmeas controle. Não foram encontradas diferenças $(P>0,05)$ nos leitões em relação as variáveis nascidos vivos, nascidos mortos $e$ mumificados. Os leitões das fêmeas que receberam dietas contendo saponinas foram mais pesados $(P<0,05)$ ao nascer $(1,2 \times 1,4 \mathrm{~kg})$ e ao desmame $(5,5 \times 5,9 \mathrm{~kg})$. Porcas alimentadas nos últimos 10 dias de gestação e na lactação com dietas contendo $160 p p m$ de saponinas têm melhor escore corporal no final da lactação e suas leitegadas são mais pesadas ao nascer e ao desmame.

Palavras-chave: extratos vegetais, leitegada, nutrição animal, suinocultura.

\section{ABSTRACT}

An experiment was carried out to study the effect of saponins on sows and their piglets performances. Thirty genetically homogeneous sows were distributed in two treatments: one control diet (CD) and a CD plus 160ppm of

\begin{abstract}
saponins. The experimental design was a completely randomized block. The studied variables in sows were feed supply, fecal characteristics (color and texture) and corporal score. In the piglets the studied variables were mummified fetuses, stillborn, born alive and total born, birth and weaning weights. Were not found differences in color in sows feces. The fecal texture for sows that received diets with saponins was about $11 \%$ more solid $(P<0.05)$. In the end of nursing the sows fed with saponins presented a corporal score $12 \%$ higher $(P<0.05)$, compared to the control females. The piglets of sows that received diets with saponins presented higher weights on birth $(1.2 \times 1.4 \mathrm{~kg})$ and at weaning $(5.5 \times 5.9 \mathrm{~kg})$ weights, both at $P<0.05$. Sows fed in the last 10 days of gestation and during the nursing period with diets containing 160ppm of saponins, had better corporal score in the end of nursing and their piglets had higher birth and weaning weights.
\end{abstract}

Key words: animal nutrition, piggery, piglets, vegetable extracts.

\section{INTRODUÇÃO}

A eficiência reprodutiva das porcas é um dos aspectos mais importantes para o sucesso da suinocultura. A gestação e a lactação são fases determinantes para melhorar essa eficiência, que é expressa em número e peso de leitões produzidos por porca anualmente. O desenvolvimento dos leitões lactentes depende da quantidade e qualidade do leite da porca. No início da lactação, essa produção está muito associada à mobilização das reservas corporais da porca (JURGENS et al., 1997), mas, à medida que a lactação avança, essa associação passa para a ingestão de alimentos.

${ }^{1}$ Departamento de Zootecnia, Universidade Federal de Santa Maria (UFSM), 97105-900, Santa Maria, RS, Brasil. E-mail: luceliah@mail.ufsm.br. Autor para correspondência.

${ }^{2}$ Departamento de Zootecnia, UFSM, Santa Maria, RS, Brasil. 
Diante da importância da alimentação para a produção de leite e desenvolvimento dos leitões, é possível melhorar o desempenho das leitegadas através da manipulação das dietas das fêmeas, especialmente no período final da gestação e na lactação. Essa manipulação pode ser protéica (DOURMAD et al., 1998), energética (COOPER et al., 2001) e utilizando nutrientes alternativos (LOWE, 1997). No entanto, outras alternativas vêm sendo apresentadas, como o uso de aditivos vegetais (AMRIK \& BILKEI, 2004), destacando-se nesse grupo as saponinas (CLINE, 1996).

As saponinas são compostos naturais encontrados em vegetais que apresentam propriedades detergentes e surfactantes. Essas propriedades são exercidas por componentes hidro e lipossolúveis. Esses componentes são constituídos de um núcleo lipofílico, tendo uma estrutura esteroidal ou triterpenoidal, com uma ou mais cadeias de carboidratos hidrossolúveis. Nas plantas, as saponinas apresentam funções como regulação do crescimento, defesa contra insetos e patógenos (OLESZEK, 1996). Essas funções revelam a importância desses compostos na adaptação e sobrevivência vegetal. Nos últimos anos, as saponinas mereceram atenção dos nutricionistas para uso na alimentação animal.

Certas plantas de deserto são especialmente ricas em saponinas (HOSTETTMANN et al., 1996). Destacam-se a Yucca schidigera, nativa dos desertos dos Estados Unidos e do México e a Quillaja saponaria, nativa de zonas áridas do Chile (WANG et al., 2000). A Quillaja saponaria é da família Rosaceae, sendo a casca e a madeira utilizadas para obtenção de saponinas. A Yucca schidigera é da família Agavaceae, sendo os galhos maduros utilizados como fonte de saponinas. Os extratos de Yucca e Quillaja estão sendo pesquisados para cães, gatos, suínos, aves, ruminantes e eqüinos. Os principais benefícios estudados são a diminuição do odor das excretas, redução da prevalência de artrite e melhora de desempenho dos animais (CHEEKE, 1996). Outra ação das saponinas é a inibição do crescimento in vitro de Escherichia coli (SEN et al., 1998). No caso específico de suínos, as saponinas reduzem a mortalidade pré e pós natal, melhoram as respostas imunológicas dos leitões quando fornecidas na dieta de porcas gestantes e lactantes (ILSLEY et al., 2005).
No entanto, os estudos dos prováveis benefícios da adição de saponinas em dietas de porcas gestantes e lactantes são limitados. Deste modo, esse trabalho teve o objetivo de avaliar o efeito da adição de fontes de saponinas em pó em dietas de porcas no final de gestação e na lactação sobre o desempenho produtivo das porcas e de suas leitegadas.

\section{MATERIAL E MÉTODOS}

O experimento foi realizado em uma granja comercial da região central do Rio Grande do Sul ( $29^{\circ} 37^{\prime} 04^{\prime}$ latitude sul e $54^{\circ} 10^{\prime} 04^{\prime \prime}$ longitude oeste), entre dezembro de 2004 e fevereiro de 2005. Foram utilizadas 30 porcas geneticamente homogêneas, de ordens de parto de um a nove. Durante o final de gestação, as porcas foram alojadas em baias coletivas, sendo transferidas para a maternidade em média oito dias antes da data provável do parto, sendo alojadas em celas individuais. O controle de temperatura dos galpões era realizado através do manejo de cortinas. As dietas (Tabela 1) foram formuladas de acordo com o NRC (NRC, 1998), sendo elaboradas com silagem de grãos úmidos de milho, soja e premix vitamínico/ mineral. Foram utilizadas quatro dietas experimentais: lactação (controle e com adição de 160ppm de fontes de saponinas) e gestação (controle e com adição de 160ppm de fontes de saponinas). As rações foram fornecidas a partir do $100^{\circ}$ dia de gestação até 21 dias de lactação. As fontes de saponinas em pó nas dietas foram obtidas de uma associação de extratos de Yucca schidigera e Quillaja saponaria.

O desenho experimental foi o de blocos ao acaso, tendo como fator de bloqueamento a ordem de parto (OP). Cada tratamento foi composto por 3 blocos e cada bloco constituído por 5 porcas, totalizando 15 unidades experimentais por tratamento. Os intervalos de OP foram divididos do seguinte modo: bloco 1: OP 1 a 3; bloco 2: OP 4 a 6; bloco 3: OP 7 a 9 . Foram avaliadas as seguintes variáveis: período pré-parto - consumo de ração, características das fezes (textura e cor diariamente); pós-parto (lactação) - consumo de ração, escore corporal (semanal), leitões (nascidos vivos, nascidos mortos, mumificados, peso ao nascer, peso ao desmame, mortalidade na lactação). As características das fezes das fêmeas avaliadas em relação a cor, variaram de 1 a 3 , onde 1 : fezes muito 
Tabela 1 - Composição centesimal e composição analisada das dietas experimentais controle nas fases de lactação e gestação ${ }^{1}$

\begin{tabular}{|c|c|c|c|c|}
\hline \multirow{3}{*}{ Ingredientes } & \multicolumn{4}{|c|}{ Dietas } \\
\hline & \multicolumn{2}{|c|}{ Gestação } & \multicolumn{2}{|c|}{ Lactação } \\
\hline & $\mathrm{T} 1$ & $\mathrm{~T} 2$ & $\mathrm{~T} 1$ & $\mathrm{~T} 2$ \\
\hline Silagem de grãos úmidos de milho & 78,55 & 78,55 & 67,6 & 67,6 \\
\hline Soja integral desativada & 17,45 & 17,45 & 28,4 & 28,4 \\
\hline Premix vitamínico $^{2}$ mineral $^{3}$ & 4,00 & 4,00 & 4,00 & 4,00 \\
\hline Saponinas, ppm & - & 160 & - & 160 \\
\hline Total & 100 & 100 & 100 & 100 \\
\hline \multicolumn{5}{|l|}{ Composição analisada } \\
\hline Matéria seca, \% & 76,24 & 76,24 & 75,71 & 75,71 \\
\hline Energia bruta, kcal/kg & 4216 & 4216 & 4324 & 4324 \\
\hline Proteína bruta, \% & 14,78 & 14,78 & 15,08 & 15,08 \\
\hline Fósforo, \% & 0,89 & 0,89 & 0,88 & 0,88 \\
\hline Cálcio, \% & 1,20 & 1,20 & 1,10 & 1,10 \\
\hline Extrato etéreo, \% & 7,98 & 7,98 & 7,65 & 7,65 \\
\hline Fibra bruta, \% & 3,84 & 3,84 & 3,82 & 3,82 \\
\hline
\end{tabular}

T1: porcas que receberam dieta controle (DC); T2: porcas que receberam DC + 160 ppm de fontes de saponinas. ${ }^{1}$ As dietas experimentais continham: Gestação: Lisina, 0,49\%; Treonina, 0,31\%; Triptofano, 0,13. Lactação: Lisina, 0,79\%; Treonina, 0,50\%; Triptofano, 0,18\%.. ${ }^{2}$ Suplemento vitamínico-mineral. Conteúdo por kg de ração: Vit. A, 228.570 UI; Vit. D3, 34.290 UI; Vit. E, 570mg; Vit. K3, 71mg; Vit. B1, 29mg; Vit. B2, 115mg; Vit. B6, 57mg; Vit. B12, 570mcg; Ác. Nicotínico, 715mg; Ác. Pantotênico, 290mg; Biotina, 1,43mg; Ác. Fólico, 17mg; Selênio, 15mg; Colina, 6.860mg; Lisina, 11.430mg; Antioxidante, 17mg; Iodo, 23mg; Cobalto, 13mg; Cobre, 2.500mg; Zinco, 2.200mg; Ferro, 2.000mg; Manganês, $1.100 \mathrm{mg}$

escuras e 3: fezes claras. Em relação a textura das fezes, havia uma variação de 1 a 3 , onde 1 : fezes duras e 3 : fezes moles. As fezes das fêmeas foram analisadas bromatologicamente somente na fase de lactação, quando as porcas permaneceram alojadas em celas individuais, proporcionando facilidade e precisão na coleta. Foi avaliado o escore das fezes dos leitões diariamente e individualmente.

Os dados foram submetidos à análise de variância ao nível de 5\% de significância, sendo utilizado o programa estatístico Minitab (MCKENZIE \& GOLDMAN, 1999). O modelo analítico incluiu tratamento $(\mathrm{T})$, intervalo de ordem de parto (OP) e interação T x OP. Em relação ao escore de fezes dos leitões, as variáveis foram submetidas à análise de variância ao nível de 5\% de significância. Foi usado como covariável o número de leitões por tratamento em cada dia de observação do escore das fezes.

\section{RESULTADOS E DISCUSSÃO}

Não foram encontradas diferenças $(P>0,05)$ entre os tratamentos para a cor das fezes. A cor predominante foi muito escura, sendo as médias 1,06 para o grupo testemunha e 1,09 para o grupo de porcas que recebeu dieta com fontes de saponinas. Para a textura das fezes, as médias foram 2,15 e 2,42 para o grupo de porcas testemunhas e para o grupo que recebeu dieta com fontes de saponinas, respectivamente. A textura das fezes apresentou diferença $(P<0,05)$, sendo que as fezes das fêmeas que receberam a dieta com adição de saponinas foram cerca de $11 \%$ mais duras. Esses resultados confirmam observações de que as fezes de cães e gatos alimentados com rações contendo extrato de Yucca schidigera são mais secas (CHEEKE, 1996). A composição analisada das fezes das porcas no período de lactação está apresentada na tabela 2 .

Em relação ao consumo de ração das fêmeas durante a gestação e lactação não foram encontradas diferenças $(\mathrm{P}>0,05)$. As médias de consumo diário de ração das porcas foram de 3,27 e 7,09kg na gestação e lactação, respectivamente. Em estudo onde houve adição de 125 ppm de extrato de Yucca na dieta de porcas lactantes ocorreu melhora no consumo de ração em 300g por dia (CLINE, 1996). Em leitões, a adição de 125ppm de Yucca na ração melhora o consumo até 28 dias de idade, mas não altera no período de 29 a 56 dias 
Tabela 2 - Composição analisada das fezes de porcas alimentadas com dietas sem e com adição de saponinas durante o período de gestação ${ }^{1}$.

\begin{tabular}{lcc}
\hline \multicolumn{2}{c}{ Tratamentos $^{2}$} \\
Composição analisada & $\mathrm{T} 1$ & $\mathrm{~T} 2$ \\
\hline Matéria seca, \% & 30,08 & 30,91 \\
Energia bruta, kcal/kg & 3751 & 3462 \\
Proteína bruta, \% & & 11,40 \\
Fósforo, \% & 11,31 & 3,71 \\
Cálcio, \% & & 7,92 \\
\hline Extrato etéreo, \% & 3,78 & 4,70 \\
\hline
\end{tabular}

${ }^{1}$ Análises realizadas no Laboratório de Nutrição Animal da Universidade Federal de Santa Maria. ${ }^{2}$ Tratamento 1: porcas que receberam dieta controle (DC); Tratamento 2: porcas que receberam $\mathrm{DC}+160 \mathrm{ppm}$ de fontes de saponinas.

de vida (YEN \& POND, 1993). No presente experimento, a ingestão na gestação não foi diferente entre os dois grupos de porcas, pois o fornecimento era restrito. No entanto, durante a lactação, a ração foi fornecida à vontade, do mesmo modo não houve diferença na ingestão, o que revela que a inclusão de saponinas na dieta não estimula nem deprime o consumo de alimento.

Os escores corporais das fêmeas no período de lactação são apresentados na tabela 3. Não foram encontradas diferenças $(\mathrm{P}>0,05)$ nas primeiras três semanas de lactação. Entretanto, na última semana, as fêmeas alimentadas com dietas contendo saponinas apresentaram um escore corporal $12 \%$ superior $(\mathrm{P}<0,05)$ às fêmeas que receberam dieta controle. Esse melhor escore pode ser explicado pelo fato de as saponinas proporcionarem aumento da permeabilidade da parede intestinal o que melhora os mecanismos da digestão e absorção (CLINE, 1996). Essa melhora foi observada na adição de 125ppm de extrato de Yucca na dieta de porcas, na qual o emagrecimento foi inferior nas porcas que consumiram a dieta contendo Yucca durante o período de lactação (CLINE, 1996). Mas se tratando de suínos em crescimento (56 dias ao abate), a adição de 125ppm de extrato de Yucca na dieta não alterou o ganho de peso (YEN \& POND, 1993).

As variáveis analisadas para os leitões são apresentadas na tabela 4. Não foram encontradas diferenças $(\mathrm{P}>0,05)$ entre os tratamentos para nascidos vivos, natimortos, mumificados e nascidos totais. Porém foram encontradas diferenças nos pesos vivos ao nascer e ao desmame $(\mathrm{P}<0,05)$. Em relação à natimortalidade e mortalidade dos leitões, dietas contendo 125 ppm de extrato de Yucca para porcas gestantes, reduziram a prevalência de natimortos e mortalidade de recém nascidos. As saponinas presentes na Yucca diminuem a amônia intestinal, reduzindo a demanda de oxigênio do tecido, permitindo maior fluxo de oxigênio aos fetos durante o nascimento. Diferente dos resultados encontrados no presente estudo, a taxa de leitegadas sem mortalidade foi de $40 \%$ para porcas que receberam dietas contendo 125ppm de Yucca e de $11 \%$ para as porcas controle (CLINE, 1996). A adição de 250ppm de extrato de Quillaja e 200 ppm de extrato de Yucca na dieta de porcas lactantes reduziu a natimortalidade e a mortalidade nas leitegadas de porcas que receberam as fontes de saponinas (ILSLEY et al., 2005). A adição de 120 ppm de pó de Yucca na dieta de porcas, do $107^{\circ}$ dia de gestação até os 21 dias

Tabela 3 - Escores corporais semanais no período de lactação de porcas alimentadas com dietas sem e com adição de saponinas $^{1}$

\begin{tabular}{|c|c|c|c|c|}
\hline \multirow{2}{*}{ Semanas } & \multicolumn{2}{|c|}{ Tratamentos } & \multirow{2}{*}{$d p r^{2}$} & \multirow{2}{*}{ Efeito } \\
\hline & $\mathrm{T} 1$ & $\mathrm{~T} 2$ & & \\
\hline 1 & 2,92 & 2,64 & 0,48 & NS \\
\hline 2 & 2,85 & 2,92 & 0,40 & NS \\
\hline 3 & 2,71 & 2,96 & 0,37 & NS \\
\hline 4 & $2,50^{\mathrm{a}}$ & $2,85^{\mathrm{b}}$ & 0,40 & $\mathrm{~T}$ \\
\hline
\end{tabular}

${ }^{1}$ resultados: 1, muito magra; 2, magra; 3, bom; 4; gorda; 5; obesa. ${ }^{2} \mathrm{dpr}$, desvio padrão residual. NS, não significativo. T, tratamento significativo ao nível de 5\%. Médias na linha seguidas de letras iguais não são diferentes. T1, dieta controle; T2, dieta com a adição de 160 ppm fontes de saponinas. 
Tabela 4 - Características de leitegadas oriundas de porcas alimentadas com dietas sem e com adição de saponinas

\begin{tabular}{|c|c|c|c|c|c|c|c|c|c|}
\hline \multirow{2}{*}{ Variáveis } & \multicolumn{2}{|c|}{ Tratamentos } & \multirow{2}{*}{$d p r^{1}$} & \multirow{2}{*}{ Efeito } & \multicolumn{3}{|c|}{ Blocos } & \multirow{2}{*}{$d p r^{1}$} & \multirow{2}{*}{ Efeito } \\
\hline & $\mathrm{T} 1$ & $\mathrm{~T} 2$ & & & 1 & 2 & 3 & & \\
\hline Nativivos, $\mathrm{n}^{\circ}$ & 11,14 & 10,35 & 3,32 & NS & 9,56 & 10,71 & 12,42 & 2,96 & NS \\
\hline Natimortos, $\mathrm{n}^{\circ}$ & 0,70 & 0,54 & 1,04 & NS & 0,30 & 1,10 & 0,66 & 0,94 & NS \\
\hline Mumificados, $\mathrm{n}^{\circ}$ & 0,56 & 0,80 & 0,88 & NS & 0,44 & 1,00 & 0,75 & 0,86 & NS \\
\hline Nascidos total, $\mathrm{n}^{\circ}$ & 12,00 & 11,36 & 3,70 & NS & $10,00^{\mathrm{a}}$ & $12,46^{\mathrm{ab}}$ & $13,83^{\mathrm{b}}$ & 3,20 & B \\
\hline Mortos, $\mathrm{n}^{\mathrm{o}}$ & 2,00 & 1,75 & 0,89 & NS & 1,50 & 1,60 & 2,11 & 0,87 & NS \\
\hline $\begin{array}{l}\text { Desmamados/porca, } \mathrm{n}^{\circ} \\
\text { Peso vivo, } \mathrm{kg}\end{array}$ & 10,21 & 10,15 & 0,94 & NS & 10,40 & 10,00 & 10,25 & 0,91 & NS \\
\hline Nascer & $1,22^{\mathrm{a}}$ & $1,36^{\mathrm{b}}$ & 0,31 & $\mathrm{~T}$ & $1,24^{\mathrm{a}}$ & $1,38^{\mathrm{b}}$ & $1,25^{\mathrm{a}}$ & 0,30 & B \\
\hline Desmame $^{2}$ & $5,53^{\mathrm{a}}$ & $5,86^{\mathrm{b}}$ & 1,15 & $\mathrm{~T}$ & 5,69 & 5,76 & 5,80 & 1,16 & NS \\
\hline
\end{tabular}

${ }^{1} \mathrm{dpr}$, desvio padrão residual. ${ }^{2}$ peso vivo ao desmame ajustado ao peso vivo ao nascer. NS, não significativo. B, bloco significativo ao nível de 5\%. T, tratamento significativo ao nível de 5\%. Médias na linha seguidas de letras iguais não são diferentes. T1, dieta controle; T2, dieta com a adição de 160 ppm de fontes de saponinas.

de lactação, reduziu a natimortalidade dos leitões (HERPIN \& CHEEKE, 2004).

No presente estudo, os pesos vivos, ao nascer e ao desmame, foram maiores $(\mathrm{P}<0,05)$ nos leitões das porcas que receberam dietas contendo saponinas. Os leitões das fêmeas que receberam dietas contendo saponinas foram mais pesados $(\mathrm{P}<0,05)$ ao nascer $(1,2 \times 1,4 \mathrm{~kg})$ e ao desmame $(5,5 \times 5,9 \mathrm{~kg})$. Os melhores resultados de peso ao nascer para os leitões das porcas que receberam a dieta com saponinas devem ser analisados com precaução, pois o período experimental durante a gestação foi muito curto. No entanto, os resultados melhores para peso ao desmame nos leitões de porcas que receberam saponinas é mais consistente e confirma observações de outros autores. Dietas contendo 125ppm de extrato de Yucca como fonte de saponinas melhoraram a taxa de crescimento de leitões na fase de creche (CROMWELL et al., 1985). A adição de 120ppm de Yucca em pó nas dietas de fêmeas lactantes melhorou a viabilidade de leitões neonatos, melhorando o ganho de peso dos leitões ao desmame (HERPIN \& CHEEKE, 2004).

Neste estudo, foram encontradas diferenças em relação aos blocos (ordem de parto). As fêmeas pertencentes ao bloco três (ordem de parto de 7 a 9$)$ apresentaram maior $(P<0,05)$ número de leitões nascidos totais em relação às fêmeas dos blocos um e dois. Mas esses resultados não foram afetados pela adição das duas fontes de saponinas na dieta. O tamanho da leitegada é menor nas ordens de parto extremas, ou seja, em primíparas e em fêmeas de ordem de parto superior a 7 (KOKETSU et al., 1997).

Os escores das fezes dos leitões medidos durante os 21 dias de lactação e no período total são apresentados na tabela 5. A ocorrência dos escores de fezes é apresentada em número médio de leitões por escore em cada tratamento. No primeiro dia, as fezes dos leitões de porcas da dieta testemunha tiveram escore 3 (fezes moles) $55 \%$ maior $(\mathrm{P}<0,05)$ que os leitões das porcas que receberam fontes de saponinas. No décimo dia, as fezes dos leitões provenientes das porcas testemunhas apresentaram ocorrência no escore 1 (fezes duras) $78 \%$ maior $(\mathrm{P}<0,05)$ que os leitões das porcas que receberam fontes de saponinas. No entanto, não houve diferença $(\mathrm{P}>0,05)$ no período total de observação entre os dois tratamentos. Diferentes resultados foram encontrados em estudo com cães e gatos. Em trabalho no qual o extrato saponificado de Yucca schidigera foi adicionado às rações de cães e gatos, concluiu-se que a Yucca auxilia no trânsito intestinal promovendo fezes mais secas (LOWE, 1997).

\section{CONCLUSÕES}

Porcas alimentadas nos últimos 10 dias de gestação e na lactação com dietas contendo 160ppm de extratos em pó como fontes de saponinas têm melhor escore corporal no final da lactação e suas leitegadas são mais pesadas ao nascer e ao desmame. 
Tabela 5 - Escore das fezes dos leitões de porcas alimentadas na lactação com dietas sem e com adição de saponinas ${ }^{1}$

\begin{tabular}{|c|c|c|c|c|c|c|c|c|c|c|c|c|}
\hline \multirow[b]{2}{*}{ Dias } & \multicolumn{4}{|c|}{ Escore 1} & \multicolumn{4}{|c|}{ Escore 2} & \multicolumn{4}{|c|}{ Escore 3} \\
\hline & $\mathrm{T} 1$ & $\mathrm{~T} 2$ & $d p r^{2}$ & Efeito & $\mathrm{T} 1$ & $\mathrm{~T} 2$ & $\mathrm{dpr}^{2}$ & Efeito & $\mathrm{T} 1$ & $\mathrm{~T} 2$ & $d p r^{2}$ & Efeito \\
\hline 01 & 4,87 & 6,25 & 2,89 & NS & 2,88 & 2,10 & 1,28 & NS & 7,63 & 4,27 & 3,49 & $\mathrm{~S}$ \\
\hline 02 & 5,58 & 6,69 & 2,75 & NS & 4,23 & 2,72 & 4,04 & NS & 2,60 & 3,60 & 2,06 & NS \\
\hline 03 & 8,78 & 8,46 & 2,50 & NS & 2,55 & 2,44 & 1,33 & NS & 1,25 & 2,33 & 1,15 & NS \\
\hline 04 & 9,28 & 9,53 & 1,94 & NS & 1,66 & 2,00 & 1,26 & NS & 2,00 & 1,00 & 1,00 & NS \\
\hline 05 & 9,57 & 9,92 & 1,25 & NS & 1,55 & 1,42 & 0,78 & NS & 0,00 & 0,00 & - & NS \\
\hline 06 & 9,50 & 9,53 & 1,50 & NS & 1,83 & 1,33 & 0,51 & NS & 1,00 & 1,33 & 0,57 & NS \\
\hline 07 & 10,14 & 10,30 & 0,63 & NS & 1,66 & 1,00 & 0,57 & NS & 0,00 & 0,00 & - & NS \\
\hline 08 & 9,78 & 10,07 & 0,86 & NS & 1,60 & 1,50 & 0,70 & NS & 1,00 & 0,00 & - & NS \\
\hline 09 & 10,21 & 10,30 & 0,63 & NS & 1,50 & 0,00 & 0,70 & NS & 1,00 & 0,00 & - & NS \\
\hline 10 & 9,64 & 7,58 & 1,15 & $\mathrm{~S}$ & 1,28 & 3,00 & 2,95 & NS & 2,00 & 3,20 & 2,16 & NS \\
\hline 11 & 9,78 & 9,76 & 0,92 & NS & 1,80 & 1,16 & 0,83 & NS & 0,00 & 0,00 & - & NS \\
\hline 12 & 9,35 & 10,07 & 0,86 & NS & 3,00 & 1,00 & 1,00 & NS & 1,25 & 1,00 & 0,50 & NS \\
\hline 13 & 9,58 & 10,00 & 1,00 & NS & 2,75 & 1,00 & 1,70 & NS & 6,00 & 1,00 & 4,58 & NS \\
\hline 14 & 9,28 & 10,07 & 1,03 & NS & 1,80 & 1,00 & 0,83 & NS & 1,66 & 1,00 & 0,67 & NS \\
\hline 15 & 9,57 & 10,15 & 0,89 & NS & 1,14 & 1,00 & 0,37 & NS & 1,00 & 0,00 & - & NS \\
\hline 16 & 9,85 & 9,83 & 0,83 & NS & 1,00 & 3,50 & 4,35 & NS & 1,00 & 1,00 & - & NS \\
\hline 17 & 9,61 & 9,76 & 0,83 & NS & 4,50 & 1,00 & 4,12 & NS & 0,00 & 0,00 & - & NS \\
\hline 18 & 9,35 & 9,69 & 1,18 & NS & 1,83 & 1,00 & 1,32 & NS & 1,00 & 2,00 & - & NS \\
\hline 19 & 8,23 & 8,83 & 1,52 & NS & 2,33 & 1,57 & 2,29 & NS & 5,00 & 4,66 & 4,00 & NS \\
\hline 20 & 8,64 & 7,50 & 3,11 & NS & 2,50 & 2,75 & 2,26 & NS & 1,00 & 2,71 & 2,87 & NS \\
\hline 21 & 8,64 & 8,69 & 1,49 & NS & 1,60 & 1,42 & 0,69 & NS & 2,00 & 1,30 & 0,51 & NS \\
\hline Média & 9,12 & 9,21 & 1,98 & NS & 2,22 & 1,97 & 1,70 & NS & 3,26 & 2,78 & 2,62 & NS \\
\hline
\end{tabular}

${ }^{1}$ Resultados: E1: Fezes duras; E2: Fezes pastosas; E3: Fezes líquidas. ${ }^{2}$ dpr, desvio padrão residual. T1, dieta controle; T2, dieta com a adição de 160 ppm de adição de fontes de saponinas. NS, não significativo; S, tratamento significativo ao nível de 5\% de significância.

\section{AGRADECIMENTOS}

Os autores agradecem à : (1) Beraca Sabará químicos e ingredientes Ltda pelo apoio financeiro e forncimento dos saponinas (produto comercial SUINATURA); (2) Granja de Suínos Toropi pelo apoio logístico de campo; (3) Conselho Nacional de Desenvolvimento Científico e Tecnológico (CNPq) pela bolsa cedida à Lucélia Hauptli; (4) à Daniela Zanforlim (divisão Feed- Beraca Sabará) pelas sugestões. e a Granja de Suínos Toropi pelo apoio na realização do experimento.

\section{REFERÊNCIAS}

AMRIK, B.; BILKEI, G. Influence of farm application of oregano on performances of sows. Can Vet J, v.45, p.674$677,2004$.

CHEEKE, P.R. Biological effects of feed and forage saponins and their impacts on animal production. Adv Exp Med Biol, v.405, p.377-385, 1996.

CLINE, J.L. et al. Effect of feeding MICRO-AID on stillbirths, preweaning mortality, blood oxygen values of piglets and blood urea nitrogen in sows. J Anim Sci, v.74 (Suppl.1), p.189, 1996. (Abstr).
COOPER, D.R. et al. Effect of energy and lysine intake in gestation on sow performance. J Anim Sci, v.79, p.23672377, 2001.

CROMWELL, G.L. et al. Efficacy of sarsaponin for weanling and growing-finishing swine housed at two animal densities. J Anim Sci, v.61(Suppl.1), p.111, 1985. (Abstr).

DOURMAD, J.Y. et al. Effect of protein and lysine supply on performance, nitrogen balance, and body composition changes of sows during lactation. J Anim Sci, v.76, p.542550, 1998.

HERPIN, P.V.A.; CHEEKE, P.R. Effect of feeding Yucca schidigera (DK powder) to the sow on piglet blood oxygenation and survival. Proc West Sect Amer Soc Anim Sci, v.55, p.145-150, 2004.

HOSTETTMANN, K. et al. Search for molluscicidal and antifungal saponins from tropical plants. Adv Exp Med Biol, v.404, p.117-128, 1996.

ILSLEY, S. E. et al. Effects of dietary quillaja saponin and curcumin on the performance and immune status of weaned piglets. J Anim Sci, v.83, p.82-88, 2005. 
JURGENS, M.H. et al. The effect of dietary active dry yeast supplement on performance of sows during gestation-lactation and their pigs. J Anim Sci, v.75, p.593-597, 1997.

KOKETSU, Y. et al. Influence of various factors on farrowing rate on farms using early weaning. J Anim Sci, v.75, p.25802587, 1997.

LOWE, J.A. The effect of Yucca schidigera extract on canine and feline faecal volatiles occurring concurrently with faecal aroma amelioration. Veterin Sci, v.63, p.67-71, 1997.

MCKENZIE, J.; GOLDMAN, R.N. The student edition of Minitab for Windows manual: release 12 . Belmont: Addison-Wesley Longman, Incorporated, 1999. 592p. (Softcover ed.).
NRC. Nutrient requirements of swine. 10 ed. Washington: National Academy, 1998. 189p.

OLESZEK, W. Alfalfa saponins: structure, biological activity, and chemotaxonomy. Adv Exp Med Biol, v.405, p.155-170, 1996.

SEN, S. et al. Effect of Quillaja saponaria saponins and Yucca schidigera plant extract on growth of Escherichia coli. Lett Appl Microbiol, v.27, p.35-38, 1998.

WANG, Y. et al. Effect of steroidal saponin from extract on ruminal microbes. J Appl Microbiol, v.88, p.887-896, 2000.

YEN, J.T.; POND, W.G. Effects of carbadox, copper, or Yucca schidigera extract on growth performance and visceral weight of young pigs. J Anim Sci, v.71, p.2140-2146, 1993. 